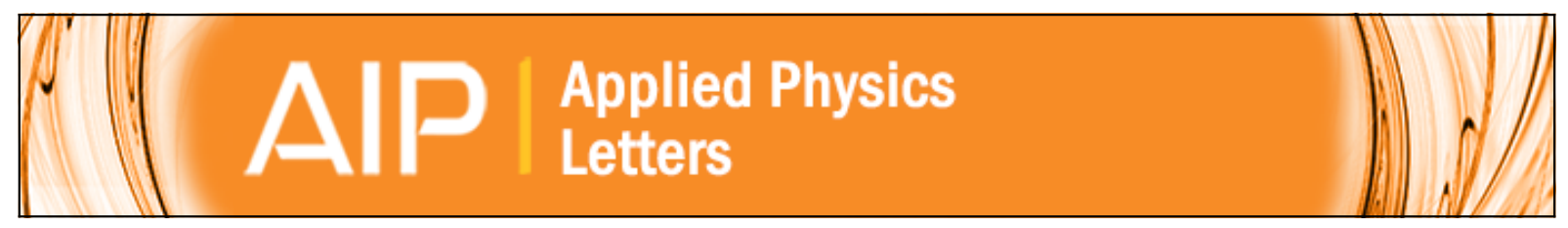

\title{
Electrical conductivity and electromagnetic interference shielding of multiwalled carbon nanotube composites containing Fe catalyst
}

H. M. Kim, K. Kim, C. Y. Lee, J. Joo, S. J. Cho, H. S. Yoon, D. A. Pejaković, J. W. Yoo, and A. J. Epstein

Citation: Applied Physics Letters 84, 589 (2004); doi: 10.1063/1.1641167

View online: http://dx.doi.org/10.1063/1.1641167

View Table of Contents: http://scitation.aip.org/content/aip/journal/apl/84/4?ver=pdfcov

Published by the AIP Publishing

\section{Articles you may be interested in}

Improved mechanical and electrical properties in electrospun polyimide/multiwalled carbon nanotubes nanofibrous composites

J. Appl. Phys. 116, 134104 (2014); 10.1063/1.4897230

Epoxy composites filled with high surface area-carbon fillers: Optimization of electromagnetic shielding, electrical, mechanical, and thermal properties

J. Appl. Phys. 114, 164304 (2013); 10.1063/1.4826529

Electrical properties of multi-walled carbon nanotube/tetrafunctional epoxy-amine composites

AIP Conf. Proc. 1459, 199 (2012); 10.1063/1.4738442

Designing of epoxy composites reinforced with carbon nanotubes grown carbon fiber fabric for improved electromagnetic interference shielding

AIP Advances 2, 022151 (2012); 10.1063/1.4730043

Super-high-frequency shielding properties of excimer-laser-synthesized-single-wall-carbonnanotubes/polyurethane nanocomposite films

J. Appl. Phys. 109, 084313 (2011); 10.1063/1.3574443

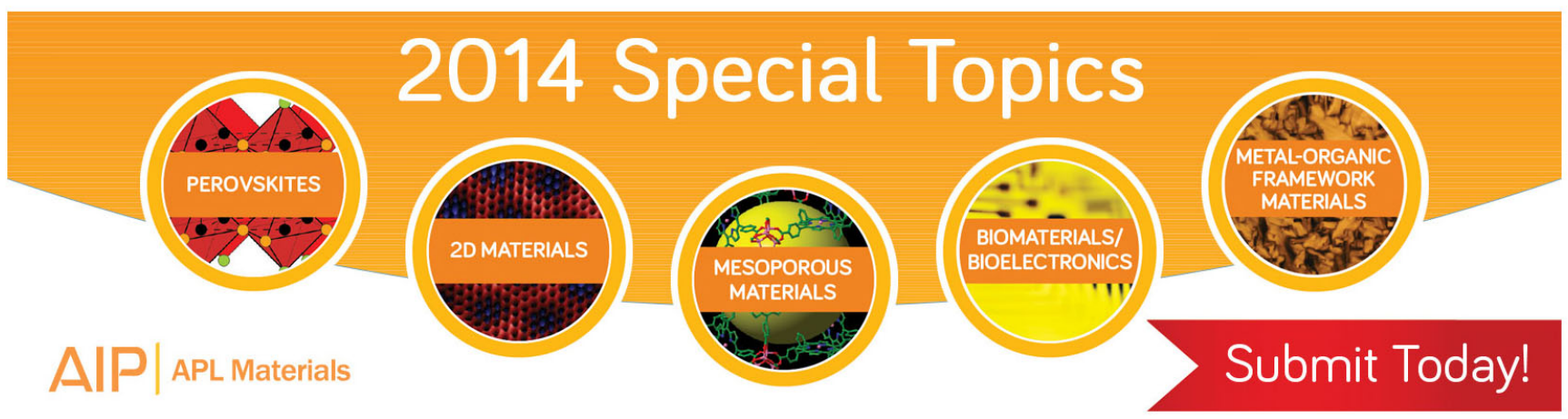




\title{
Electrical conductivity and electromagnetic interference shielding of multiwalled carbon nanotube composites containing Fe catalyst
}

\author{
H. M. Kim, K. Kim, C. Y. Lee, and J. Joo ${ }^{\text {a) }}$ \\ Department of Physics, Korea University, Seoul 136-701, Korea \\ S. J. Cho \\ Iljin Nanotech Co., Ltd., Kangseo-Ku, Seoul 157-810, Korea \\ H. S. Yoon \\ NOVATEMS Inc., Manan-Gu, Anyang-City, Kyunggi-Do 430-817, Korea \\ D. A. Pejaković, J. W. Yoo, and A. J. Epstein \\ Department of Physics, the Ohio State University, Columbus, Ohio 43210
}

(Received 7 October 2002; accepted 20 November 2003)

\begin{abstract}
Thin and flexible composite films of raw or purified multiwalled carbon nanotube (MWCNT) with various mass fractions and poly(methylmethacrylate) (PMMA) were synthesized for electromagnetic interference (EMI) shielding material. From scanning electron microscopy and high-resolution transmission electron microscopy photographs, we observed the formation of a conducting network through MWCNTs in an insulating PMMA matrix and the existence of an $\mathrm{Fe}$ catalyst in MWCNTs. The dc conductivity $\left(\sigma_{\mathrm{dc}}\right.$ ) of the systems increased with increasing MWCNT mass fraction, showing typical percolation behavior. The measured EMI shielding efficiency (SE) of MWCNT-PMMA composites by using the extended ASTM D4935-99 method (50 MHz-13.5 $\mathrm{GHz}$ ) increased with increasing MWCNT mass fraction as $\sigma_{\mathrm{dc}}$. The highest EMI SE for raw MWCNT-PMMA composites was $\sim 27 \mathrm{~dB}$, indicating commercial use for far-field EMI shielding. The contribution of absorption to total EMI SE of the systems is larger than that of reflection. Based on magnetic permeability, we suggest raw MWCNTs and their composites can be used for near-field EMI shielding. (C) 2004 American Institute of Physics. [DOI: 10.1063/1.1641167]
\end{abstract}

Singlewalled and multiwalled carbon nanotubes (CNTs) have been extensively studied in both fundamental and applied research fields. ${ }^{1}$ The CNT has been known as a nanomaterial with a well-defined structure. ${ }^{2}$ Nanotips for field emission display and for scanning probe microscopy, field effect transistor, supercapacitor, and hydrogen storage material have been suggested and studied for applications of CNTs. ${ }^{3}$ Multiwalled carbon nanotubes (MWCNTs) which require purification for characterization have been used in commercial applications, because of easy mass production through the chemical vapor deposition method. ${ }^{4}$ Electromagnetic interference (EMI) shielding is one of the promising applications by using conducting MWCNTs and their composite.

EMI shielding in near field $(k r \ll 1$, where $k$ is the wave number and $r$ is the distance from the EM source to detector) and far field $(k r \gg 1)$ ranges has been important for the enhancement of efficiency and the lifetime of electrical devices and equipment. In general, electrically conducting materials such as typical metals, graphite, and conducting polymers have been used for EMI shielding in far field regions. ${ }^{5}$ Polymer-based conducting systems have been considered as functional EMI shielding materials, because of easy synthesis, flexibility, light weight, low cost, mass production, etc. ${ }^{6}$ The absorption of shielding material in mobile electrical equipment having difficulty with electrical grounding has been emphasized for effective EMI shielding.

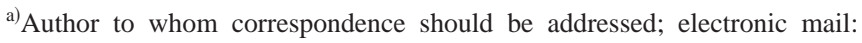
jjoo@korea.ac.kr
}

In this study, we synthesized thin and flexible films of MWCNT and poly(methylmethacrylate) (PMMA) composites for EMI shielding materials. The structural properties of the systems were investigated through scanning electron microscopy (SEM), high-resolution transmission electron microscopy (HR-TEM), and x-ray photoelectron spectroscopy (XPS) experiments. From dc conductivity $\left(\sigma_{\mathrm{dc}}\right)$ and its temperature dependence, the percolation characteristics as a function of mass fraction of MWCNTs in PMMA were observed. Based on the EMI shielding efficiency (SE), MWCNT composites are promising absorption materials for EMI shielding in far field ranges.

The raw MWCNTs provided by Iljin Nanotech Co., Ltd., were synthesized by chemical vapor deposition method using $\mathrm{Fe}$ as the main catalyst. ${ }^{4}$ Raw MWCNTs were purified by oxidation at $555{ }^{\circ} \mathrm{C}$ in air followed by $\mathrm{HCl}$ treatment for 24 h. SEM, HR-TEM, and energy dispersive x-ray spectroscopy (EDX) were used for the structure of MWCNTs and the confirmation of purification. For thin and flexible film, raw or purified MWCNTs were dispersed in toluene with PMMA through stirring and sonication for $24 \mathrm{~h}$. The weight percent of MWCNTs varied from 0.1 to $40 \mathrm{wt} \%$. The dispersed solution was cast onto a glass substrate and dried in vacuum oven at $40^{\circ} \mathrm{C}$. The thickness of the film was from $\sim 60$ to $\sim 165 \mu \mathrm{m}$. For $\sigma_{\mathrm{dc}}$ measurements, the four-probe method was used to eliminate contact resistance and a Janis closecycle refrigerator system was used for temperature dependence of resistivity $\left[\rho(T) \equiv 1 / \sigma_{\mathrm{dc}}(T)\right]$. A HP8719ES vector network analyzer with an S-parameter set was used to measure frequency dependence of EMI SE based on the ASTM 

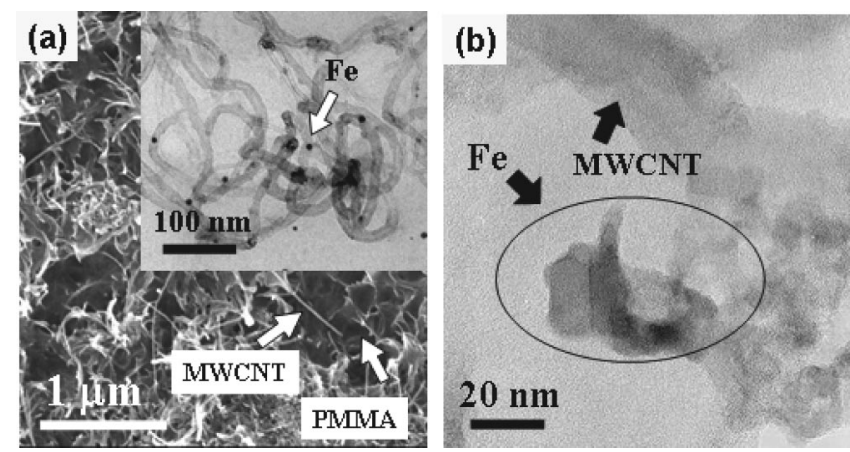

FIG. 1. (a) SEM image of the cross section of $12 \mathrm{wt} \%$ of raw MWCNTPMMA composite film. Inset: HR-TEM image of the Fe particles inside raw MWCNTs. (b) HR-TEM image of Fe metal cluster outside purified MWCNTs.

D4935-99 method. ${ }^{7,8}$ Magnetic permeability was measured by using an ac susceptometer and superconducting quantum interference device (SQUID).

Figure 1(a) shows SEM image of the cross section of 12 wt $\%$ of raw MWCNT-PMMA composite films. We observed MWCNT networks within an insulating PMMA background. All of the raw MWCNT and purified MWCNT composite films showed similar structures. We analyzed that these MWCNT networks with Fe particles play important roles in improvement of electrical conductivity and EMI SE in percolation phenomena. The inset of Fig. 1(a) presents HR-TEM image of an Fe catalyst (average diameter is $\sim 7$ $\mathrm{nm}$ ) inside raw MWCNTs, which were also reported earlier by other groups. ${ }^{4,9}$ As shown in Fig. 1(b) of the HR-TEM image for purified MWCNTs, most of the Fe catalyst particles were removed through $\mathrm{HCl}$ treatment, whereas dissolved Fe particles were formed into clusters and attached to MWCNTs, resulting from EDX analysis. From XPS wide scan of purified MWCNTs, the atomic percent ratio of Fe to $\mathrm{C}$ of purified MWCNTs is $\sim 0.75 \%$, which implies that the relatively small amount of $\mathrm{Fe}$ clusters were still remained after purification. From the results of Ar ion sputtering XPS experiments with various sputtering times, we observed that the distribution of Fe clusters in purified MWCNTs was homogeneous.

Figure 2 shows $\sigma_{\mathrm{dc}}(\mathrm{RT})$ of raw and purified PMMAMWCNT composites as a function of mass fraction $(p)$ of MWCNTs in logarithmic scale. The results show that $\sigma_{\mathrm{dc}}(p)$ of raw MWCNT-PMMA composites follows the scaling law of percolation theory described as $\sigma(p) \propto\left|p-p_{c}\right|^{t}$. The slope, i.e., the critical exponent $t$ in Fig. 2 changed from 12.39 to 2.15 , implying a percolation threshold at $p$ $\sim 0.003$. For three-dimensional system, the percolation threshold is $f_{c} \approx 0.16$ by volume fraction. ${ }^{10}$ The relatively small value of the percolation threshold observed in the MWCNT systems is accounted for MWCNTs as filler material having a one-dimensional large aspect ratio. For the composites of purified MWCNTs and PMMA, the critical exponent $t$ is $\sim 5.31$. It is expected that the exponent $t$ could be also changed for purified MWCNT-PMMA composites at a lower mass fraction of purified MWCNTs. The difference in the values of $t, \sigma_{\mathrm{dc}}$, and $\sigma_{\mathrm{dc}}(p)$ between raw and purified MWCNT-PMMA composites mainly originates from the remaining $\mathrm{Fe}$ catalyst particles and amorphous carbons in raw

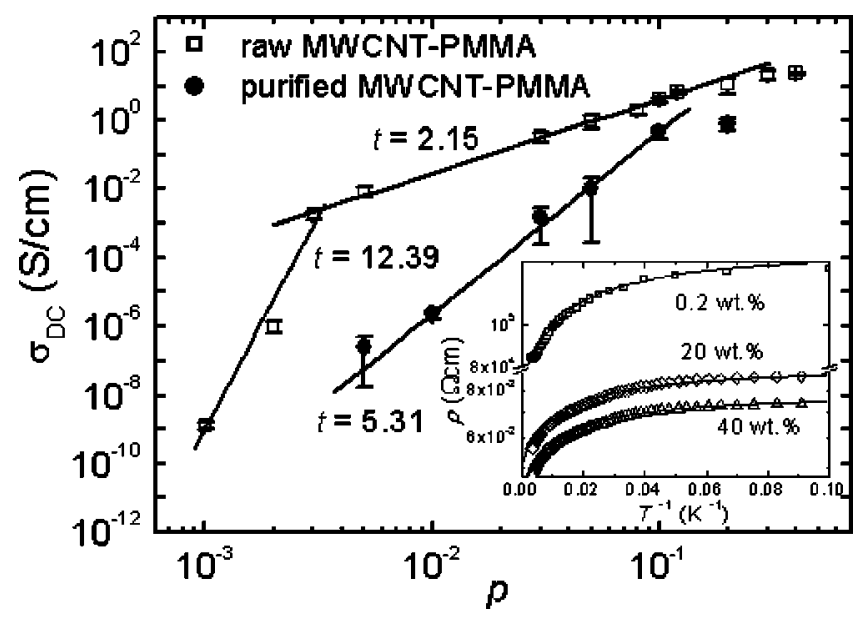

FIG. 2. dc conductivity ( $\left.\sigma_{\mathrm{dc}}\right)$ vs mass fraction $(p)$ of raw (open markers) and purified (solid markers) MWCNT-PMMA composites at RT. Inset: Temperature dependence of resistivity $[\rho(T)]$ of $0.2,20$, and 40 wt $\%$ raw MWCNT-PMMA composites. The solid lines in the inset are based on the Sheng's model.

MWCNTs and the surface or structural damage of MWCNTs during purification. The inset of Fig. 2 shows the $\rho(T)$ of the raw MWCNT-PMMA composites. The $\rho(T)$ of raw MWCNT-PMMA composites follows Sheng's tunneling conduction model, ${ }^{11}$ described as $\rho(T) \propto \exp \left[T_{1} /\left(T+T_{0}\right)\right]$, where $T_{1}$ is the activation energy and $T_{0}$ is related to temperature independent conductivity. The measured $T_{1}$ and $T_{0}$ obtained from Fig. 2 are in the range of $43-105 \mathrm{~K}$ and $72-$ $214 \mathrm{~K}$, respectively, depending on the concentration of MWCNTs in PMMA materials. The raw MWCNT-PMMA composites consist of metallic islands due to the aggregation of amorphous carbons, conducting MWCNTs, and Fe particles and insulating PMMA, which induce charge tunneling between metallic islands.

The $\mathrm{SE}_{\text {total }}$ is defined in terms of the ratio of the power of the incident and transmitted $\mathrm{EM}$ wave as $\mathrm{SE}_{\text {total }}$ $\equiv 10 \log \left(P_{I} / P_{T}\right)=20 \log \left(E_{I} / E_{T}\right)$, where $P_{I}\left(E_{I}\right)$ and $P_{T}\left(E_{T}\right)$ are power (electric field) of incident and transmitted EM waves, respectively. ${ }^{5,6}$ The unit of the EMI SE is decibel. Figure 3(a) represents the $\mathrm{SE}_{\text {total }}$ of raw and purified MWCNT-PMMA composites as a function of frequency (50
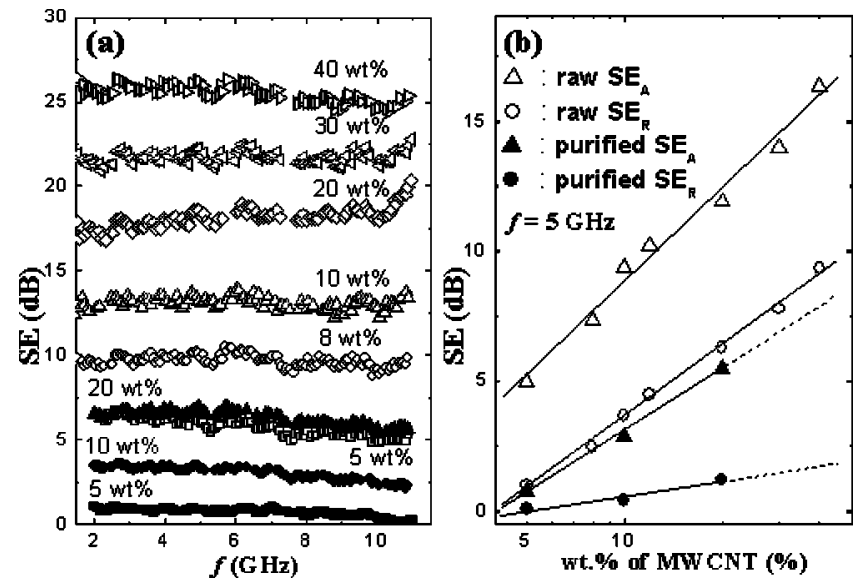

FIG. 3. EMI SE of (a) raw (open markers) and purified (solid markers) MWCNT-PMMA composites as a function of frequency and (b) comparison of $\mathrm{SE}_{A}$ and $\mathrm{SE}_{R}$ of MWCNT-PMMA composites as a function of mass fraction of MWCNTs at $f=5 \mathrm{GHz}$. 


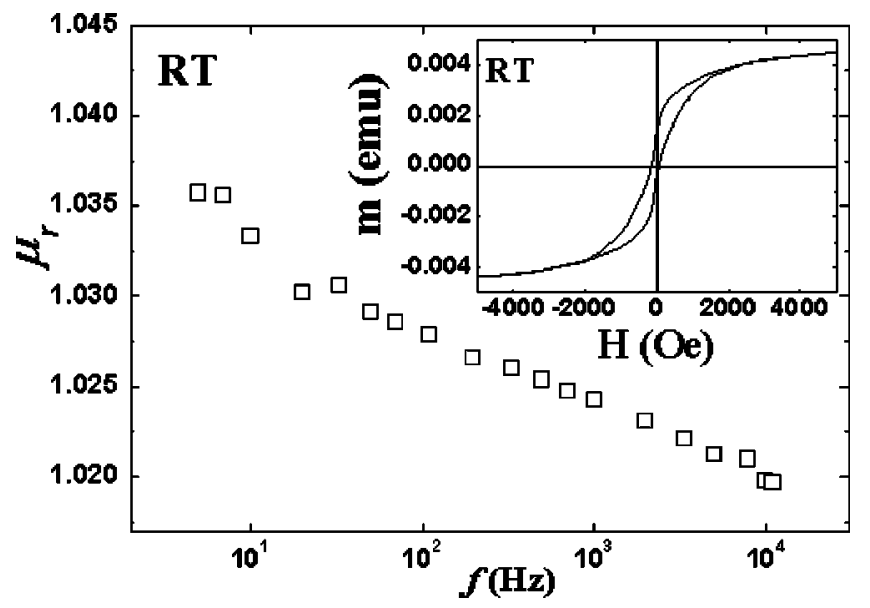

FIG. 4. Frequency dependence of permeability $\left(\mu_{r}\right)$ for raw MWCNTs at RT. Inset: Hysteresis of raw MWCNT samples at RT.

$\mathrm{MHz}-13.5 \mathrm{GHz}$ ). The measured EMI SEs of the systems are almost independent of frequency. The $\mathrm{SE}_{\text {total }}$ increases as the mass fraction of raw and purified MWCNTs increases, as shown in Fig. 3(a). The $\mathrm{SE}_{\text {total }}$ increases as $\sigma_{\mathrm{dc}}$ of shielding material increases based on the EMI shielding theory. The $\mathrm{SE}_{\text {total }}$ of raw MWCNT (40 wt \%)-PMMA composite is measured to be $\sim 27 \mathrm{~dB}$, indicating possible use for commercial applications. ${ }^{6}$ The $\mathrm{SE}_{\text {total }}$ of the composite with 20 wt \% purified MWCNTs is $\sim 7 \mathrm{~dB}$ in the measured frequency range. The increment of the EMI SE in composite films is due to the conductivity increment from the network formation of conducting MWCNTs in an insulating PMMA background. The higher EMI SE of raw MWCNT-PMMA composites compared to that of purified ones mainly originates from the Fe metal catalyst in the raw MWCNTs. The relation between transmittance $(T)$, reflectance $(R)$, and absorbance (A) through shielding material is described as $T+R+A$ $=1$. The $\mathrm{SE}_{\text {total }}$ is the summation of the $\mathrm{SE}$ due to absorption $\left(\mathrm{SE}_{A}\right)$, reflection $\left(\mathrm{SE}_{R}\right)$, and multiple replection $\left(\mathrm{SE}_{M}\right)$, i.e., $\mathrm{SE}_{\text {total }}=\mathrm{SE}_{A}+\mathrm{SE}_{R}+\mathrm{SE}_{M} \cdot{ }^{5,6}$ The transmittance $T$ is measured from the ratio of $P_{T}$ to $P_{I}$, i.e., $T=\left(P_{T} / P_{I}\right)$ $=\left(E_{T} / E_{I}\right)^{2}$. Thus, the $\mathrm{SE}_{\text {total }}$ of shielding material can be written as $\mathrm{SE}_{\text {total }}=20 \log \left(E_{I} / E_{T}\right)=-10 \log T$. Considering the effective absorbance $\left(A_{\text {eff }}\right)$, which is defined as $A_{\text {eff }} \equiv(1$ $-R-T) /(1-R)$ with respect to the power of the incident EM wave inside the shielding material, the $S E$ due to reflectance and effective absorbance can be described as ${ }^{8} \mathrm{SE}_{R} \simeq$ $-10 \log (1-R)$ and $\mathrm{SE}_{A} \simeq-10 \log [T /(1-R)]$. Figure 3(b) compares $\mathrm{SE}_{A}$ and $\mathrm{SE}_{R}$ for raw and purified MWCNTPMMA composites. As the MWCNT mass fraction increases, both $\mathrm{SE}_{A}$ and $\mathrm{SE}_{R}$ increase. The contribution of absorption to the total EMI SE is larger than the reflection for the systems, because of moderate conductivity of the composites. The results suggest that the systems are promising EMI absorption materials in $\mathrm{rf}$ and microwave frequency ranges. We analyze that the MWCNT networks as a conducting path play an important role for $\sigma_{\mathrm{dc}}$ and EMI SE as shown in Fig. 1(a), not Fe catalyst particles, because the concentration of Fe catalyst particles or clusters is too low.

Figure 4 represents the frequency dependence of the real part of permeability $\mu_{r}(f)$ measured by an ac susceptometer at RT. ${ }^{12}$ Initial de permeability $\left(\mu_{r}\right)$ of raw MWCNTs is measured to be $\sim 1.054$, obtained from the slope of hysteresis through SQUID experiments at RT, as shown in the inset of Fig. 4. The coercivity $\left(H_{c}\right)$ of the raw MWCNTs is $\sim 100$ Oe at RT $(\sim 2210 \mathrm{Oe}$ at $5 \mathrm{~K})$, while that of the purified MWCNTs is reduced to $\sim 50$ Oe at RT $(\sim 770$ Oe at $5 \mathrm{~K})$. The magnetic permeability of raw MWCNT samples originates from the ferromagnetic $\mathrm{Fe}$ metal catalyst inside the tubes as shown in Fig. 1. The measured permeability decreases with increasing frequency. In near-field shielding theory, the EMI SE due to absorption is described as $\mathrm{SE}_{A}$ $\propto\left(f \mu_{r} \sigma\right)^{1 / 2}$. $^{5}$ When multiple reflections are negligible, the $\mathrm{SE}_{\text {total }}$ increases due to $\mathrm{SE}_{A}$ as $\mu_{r}$ increases in the near-field range. We propose that raw MWCNTs and their composites are promising EMI shielding materials in the near-field range, because of the magnetic permeability from Fe catalysts inside MWCNTs.

In conclusion, we synthesized MWCNT-PMMA composites for EMI shielding. The $\sigma_{\mathrm{dc}}$ and EMI SE of MWCNT-PMMA composites increase with increasing MWCNT mass fraction at RT. We observed the percolation behavior with the threshold $p_{c} \simeq 0.003$ for raw MWCNTPMMA composites. The EMI SE of the composites with 40 wt $\%$ MWCNTs is $\sim 27 \mathrm{~dB}$. The contribution of absorption to total EMI SE for both raw and purified MWCNT-PMMA composites is larger than that of the reflection, suggesting that the systems are promising EMI absorption materials. Because of the magnetic permeability of raw MWCNTs with an Fe metal catalyst, the systems can be used for EMI shielding material in the near-field range.

This work was supported by the IMT-2000 Project, Ministry of Commerce, Industry, and Energy, Korea, the Brain Korea 21, and the US Office of Naval Research.

${ }^{1}$ Science and Application of Nanotubes, edited by D. Tománek and R. J. Enbody (Kluwer Academic/Plenum, New York, 2000); R. Saito, G. Dresselhaus, and M. S. Dresselhaus, Physical Properties of Carbon Nanotubes (Imperial College Press, London, 1998).

${ }^{2}$ S. Iijima, Nature (London) 354, 56 (1991); Y. Ando, X. Zhao, and H. Shinoyama, Carbon 39, 569 (2001).

${ }^{3}$ L. Dai and A. W. H. Mau, Adv. Mater. (Weinheim, Ger.) 13, 899 (2001); Q. H. Wang, A. A. Setlur, J. M. Lauerhaas, J. Y. Dai, E. W. Seelig, and R. P. H. Chang, Appl. Phys. Lett. 72, 2912 (1998).

${ }^{4}$ P. Pinheiro, M. C. Schouler, P. Gadelle, M. Mermoux, and E. Dooryhée, Carbon 38, 1469 (2000); K. Hernadi, Chem. Phys. Lett. 363, 169 (2002).

${ }^{5} \mathrm{H}$. W. Ott, Noise Reduction Techniques in Electronic Systems, 2nd ed. (Wiley, New York, 1998).

${ }^{6}$ J. Joo and A. J. Epstein, Appl. Phys. Lett. 65, 2278 (1994); J. Joo and C. Y. Lee, J. Appl. Phys. 88, 513 (2000).

${ }^{7}$ ASTM D4935-99, Standard Test Method for Measuring the Electromagnetic Shielding Effectiveness of Planar Materials, 1999.

${ }^{8}$ Y. K. Hong, C. Y. Lee, C. K. Jeong, D. E. Lee, K. Kim, and J. Joo, Rev. Sci. Instrum. 74, 1098 (2003)

${ }^{9}$ K. Hernadi, A. Fonseca, J. B. Nagy, D. Bernaerts, J. Riga, and A. Lucas, Synth. Met. 77, 31 (1996); R. Andrews, D. Jacques, D. Quian, and E. C. Dickey, Carbon 39, 1681 (2001).

${ }^{10}$ D. Stauffer and A. Aharony, Introduction to Percolation Theory, 2nd ed. (Taylor \& Francis, London, 1992); R. Zallen, The Physics of Amorphous Solids (Wiley, New York, 1983).

${ }^{11}$ P. Sheng, E. K. Sichel, and J. I. Gittleman, Phys. Rev. Lett. 40, 1197 (1978).

${ }^{12}$ G. Asti and M. Solzi, J. Magn. Magn. Mater. 157-158, 559 (1996). 